\title{
Erstversorgung bei Pferden mit Frakturverdacht
}

\author{
Bianca Carstanjen, Stefan Rattenhuber
}

Muskuloskeletale Verletzungen stellen eine häufige Notfallindikation für den Pferdetierarzt dar. Neben einer fachgerechten Stabilisierung der frakturierten Gliedmaße steht der stress- und schmerzreduzierte Transport des Frakturpatienten in eine geeignete Klinik im Vordergrund. Einfache, jedoch zwingend notwendige Maßnahmen wie die Stabilisierung der Gliedmaße mit der Möglichkeit der Gewichtaufnahme haben auf den weiteren Krankheitsverlauf, die Therapie und die Prognose des Frakturpatienten wesentlichen Einfluss. Hier sind die Vermeidung einer Frakturdislokation und die Schonung des Weichteilgewebes hervorzuheben. In diesem Beitrag werden die Untersuchung des Frakturpferds, die fachgerechte Stabilisierung von Frakturen sowie der Transport des Frakturpatienten in eine Klinik beschrieben.

\section{Einleitung}

Der umfassenden Erstversorgung von Frakturverletzungen beim Pferd kommt eine besondere Rolle zu, da der Einfluss auf den Behandlungserfolg entscheidend ist und mitunter sogar über Leben und Tod entscheiden kann. Eine Vielzahl von Frakturen der Gliedmaße ist, abhängig von Frakturlokalisation und Konfiguration, in Spezialkliniken mit akzeptablen Erfolgen therapierbar $[4,8,12]$. Dem überweisenden praktischen Tierarzt kommt eine Schlüsselrolle zu, da eine unsachgemäße Erstversorgung und ein falscher Transport des Frakturpferds eine erfolgversprechende Therapiemöglichkeit zunichtemacht [7]. Die Notfallversorgung des Frakturpatienten sollte zum Ziel haben, weitere Schäden an der verletzten Gliedmaße zu vermeiden und dem Pferd einen sicheren, möglichst schmerz- und stressfreien Transport in die Klinik zu ermöglichen ( $\triangleright$ Kasten $\mathbf{1})$.

\section{Erste-Hilfe-Checkliste}

1. Ruhige Umgebung um den Frakturpatienten

2. Allgemeine Untersuchung; Überprüfung der Vitalparameter

3. Unkooperative/schmerzhafte Patienten sollten, wenn möglich, mittels Zwangsmaßnahmen (Nasenbremse etc.) ruhig gestellt werden

4. Spezielle Untersuchung der frakturierten Gliedmaße

5. Fachgerechte Stabilisation der verletzten Gliedmaße mittels Schienenverband oder Cast-Verband

6. Fachgerechter Transport des Frakturpatienten zu einer Spezialklinik. Die Klinik muss vor dem Transport informiert werden. Evtl. muss der Frakturpatient (Schock etc.) vor dem Transport stabilisiert werden. Nicht behandelbare Frakturpatienten werden vor Ort euthanasiert.

\section{Untersuchung bei Frakturverdacht}

Der Frakturpatient sollte in ruhiger Atmosphäre gründlich untersucht werden [11]. Bei Wettkämpfen sollten Schaulustige ferngehalten werden. Liegende Pferde sind möglichst vor einem ersten Aufstehversuch zu untersuchen [6]. Die Allgemeinuntersuchung gibt Aufschluss über die Vitalparameter des Pferdes und beinhaltet unter anderem die Beurteilung von Körpertemperatur, Herz- und Atemfrequenz, Schleimhäuten und Hauttugor. Todesfälle durch frakturassoziierten Blutverlust und Erschöpfungszustände sind beschrieben $[10,11]$.

Der Allgemeinuntersuchung schließt sich die spezielle Untersuchung des Bewegungsapparats an. Bei unkooperativen, schmerzhaften Frakturpatienten reicht das Anlegen einer Nasenbremse meist aus, um die verletzte Region der Gliedmaße untersuchen zu können [7]. Der Bereich der Fraktur wird auf Hautverletzungen und Weichteilschwellungen hin untersucht, da diese die Prognose beeinflussen können. Meist kann aufgrund der veränderten anatomischen Gegebenheiten, dem klinischen Bild, d.h. akute, hochgradige Lahmheit, eine Verdachtsdiagnose gestellt werden.

In unklaren Fällen wird das Pferd so lange als Frakturpatient betrachtet, bis die Verdachtsdiagnose widerlegt werden kann.

\section{Wundversorgung}

Bei Frakturen und Frakturverdacht hat die Notfallstabilisierung oberste Priorität, um das Risiko eines Weichteilschadens und einer Frakturdislokation einzugrenzen. Wunden werden gesäubert und provisorisch versorgt.

\section{Infektionsprophylaxe}

Bei offenen Frakturen oder stark traumatisiertem Weichteilgewebe sollten systemische Breitspektrum-Antibiotika (z. B. Penicillin und Gentamycin) appliziert werden. Der Tetanusschutz des Pferdes muss gewährleistet sein. Bei unbekanntem Impfstatus sollte Tetanus-Antitoxin verabreicht werden.

\section{Bildgebende Verfahren}

Bei nicht stabilen Frakturen sollte das Pferd erst nach äußerer Stabilisierung der Fraktur geröntgt werden. Eine stabilisierte Gliedmaße ist einfacher zu röntgen und Holzlatten bzw. Polyvinylchlorid-(PVC)Schienen sind röntgendurchlässig. Ein Ablegen des Pferdes unter Vollnarkose zur Erstellung von Röntgenbildern ist kontraindiziert. Bei Frakturverdacht unter Feldbedingungen, ohne Möglichkeit des Röntgens, sollte eine fachgerechte Stabilisierung der Gliedmaße vorgenommen werden. Das Pferd sollte dann zur Diagnostik und eventuellen Therapie an eine Klinik überwiesen werden.

\section{Schmerztherapie}

Eine adäquate Stabilisierung der Fraktur verringert Angst und Schmerzen des Patienten. Nichtsdestotrotz ist die Verabreichung von Analgetika und Antiphlogisti- 


\section{ps.fachspiegel}
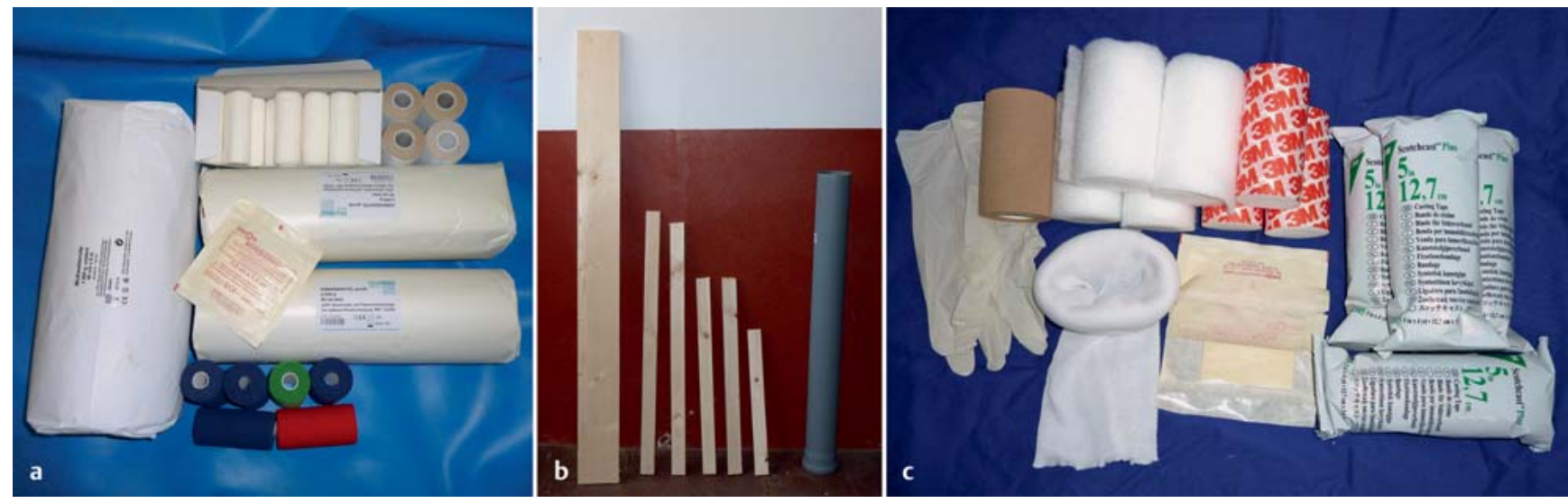

Abb. 1 a bis c Materialien zur (a) Immobilisierung bzw. (b, c) Stabilisierung einer Gliedmaße. a Verbandmaterialien zum Anlegen eines Robert-Jones-Verbands. b Verschiedene Hilfsmittel zur Stabilisierung von Frakturen der Gliedmaße, d. h. Holzlatten, PVG-Schienen. c Materialien zum Anlegen eines Cast-Verbands im Bereich der distalen Gliedmaße.
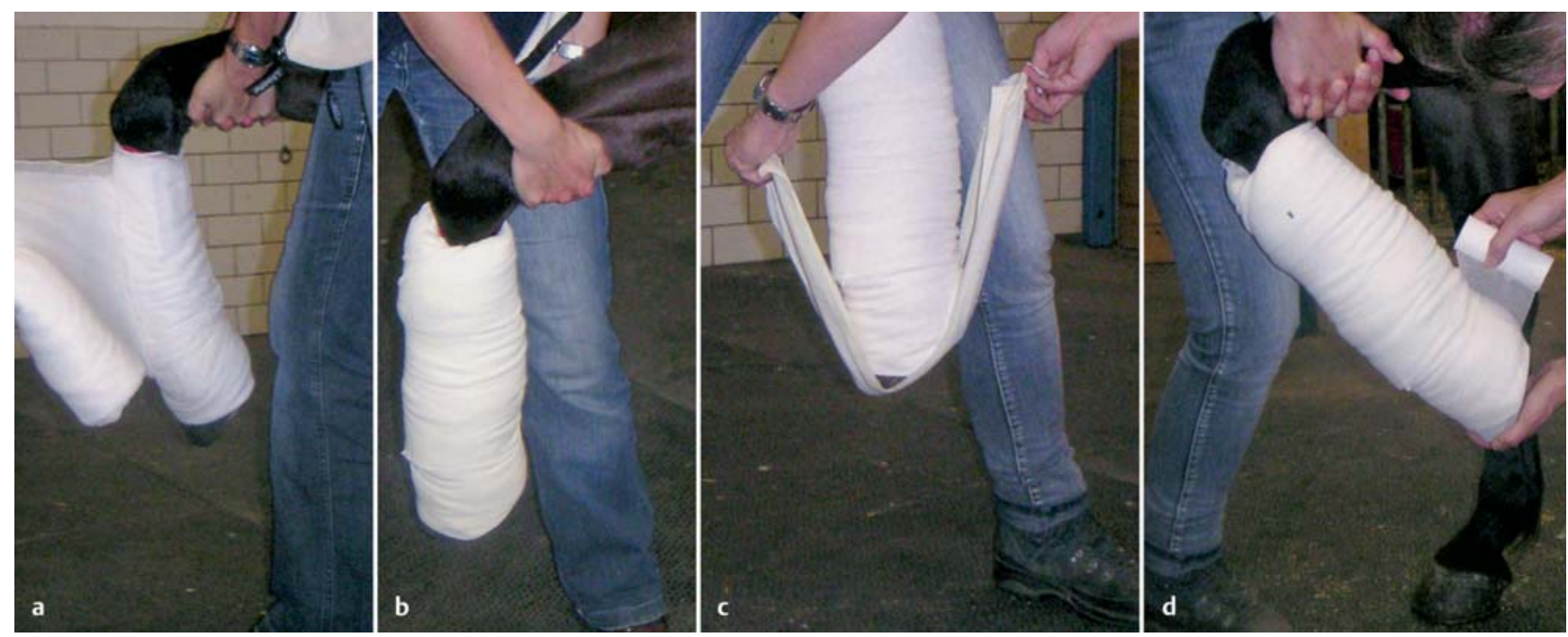

Abb. 2 a bis d Notfall-Castverband im Bereich der distalen Vordergliedmaße. Das Vorderbein wird im Bereich des distalen Radius aufgehoben, wobei die distale Gliedmaße in gerader Linie herunterhängt. Bei geschlossenen Frakturen eignet sich ein Zinkleimverband als erste stabilisierende Verbandsschicht. a, b Die weitere Verbandsschicht besteht aus Castverbandwatte oder Unterlegschaum-Bandage. c Die Castbinde bedeckt die Hufsohle (hier kann ein Hufkeil bzw. können Castbinden im Bereich der Trachten angebracht werden). d Darauf folgt ein Anlegen des Castverbands im Bereich der distalen Gliedmaße.

ka, wie nicht steroidale Antiphlogistika, im Anschluss an die bzw. vor der Frakturstabilisierung angezeigt.

\section{Prognose}

Frakturen der proximalen Gliedmaße sind bei Pferden mit einem Gewicht über 300 kg meist schwierig zu therapieren [11].

Komplikationen sind unter anderem durch das erhöhte Gewicht der Tiere und die damit erhöhte Krafteinwirkung auf die entsprechenden Knochen bedingt. Die chirurgische Versorgung von Pferden mit einem Gewicht unter $300 \mathrm{~kg}$ hat, abhängig von Frakturtyp und Lokalisation, eine ausreichend gute Prognose $[8,11]$. Aussichtslose Fälle sollten jedoch nicht mehr transportiert werden. Diese Frakturpatienten sollen aus Gründen des Tierschutzes am bzw. nahe des Unfallorts euthanasiert werden [5]. Die Besitzer des Pferdes sollten darauf hingewiesen werden, dass eine offizielle pathologische Untersuchung bei einigen Haftpflicht- und Versicherungsfragen notwendig sein kann.

\section{Unterschiedliche Verbandsverfahren}

Als Grundsatz bei der Frakturstabilisierung muss das Prinzip angewendet werden, dass jeweils die der Fraktur benachbarten Gelenke mit in den Verband eingeschlossen werden sollten, um eine ausreichende Ruhigstellung der betroffenen Lokalisation zu gewährleisten. Der Robert-JonesVerband - d.h. ein Verband mit einem Durchmesser, der mindestens dem 3-fa- chen Durchmesser der Gliedmaße entspricht - dient der Immobilisierung einer Gliedmaße ( Abb. 1 a, Tab. 1) [3]

Eine ausreichende Stabilisierung einer gebrochenen Gliedmaße kann jedoch mittels Robert-Jones-Verband nicht erreicht werden. Zu diesem Zweck müssen widerstandsfähige Materialien eingesetzt werden $(\checkmark$ Abb. $1 \mathbf{b}, \mathbf{c})$. Zugeschnittene PVCSchienen sowie Holzlatten oder Metallstäbe sind stabiles, kostengünstiges, aber schlecht modellierbares Schienenmaterial ( $\downarrow$ Abb. 1b). Abhängig von der betroffenen Gliedmaße und Lokalisation der Fraktur werden die Schienen meist im $90^{\circ}$-Winkel zueinander, d.h. kranial oder kaudal und lateral mittels nicht elastischen Klebebands über einem Verband befestigt. Nicht fachgerecht durchgeführte Stabilisierungsmaßnahmen können zum Verrut- 
- Tab. 1 Benötigtes Material zum Anlegen eines Robert-Jones-Verbands bzw. Cast-Verbands im Bereich der distalen Gliedmaße und im Bereich der gesamten Gliedmaße.

\begin{tabular}{|c|c|c|}
\hline & Material & Material \\
\hline & Robert-Jones-Verband & Fiberglas-Cast \\
\hline \multirow{5}{*}{$\begin{array}{l}\text { distale } \\
\text { Gliedmaße }\end{array}$} & 3-4 Watterollen & Cast-Foam/synthetische Cast-Watte \\
\hline & 10 elastische Bandagen & $5-6$ Cast-Binden $(12,5 \mathrm{~cm})$ \\
\hline & 3 elastische adhäsive Bandagen & 1-2 Bandagen \\
\hline & Klebeband & 1 elastische adhäsive Bandage \\
\hline & & Klebeband \\
\hline \multirow{6}{*}{$\begin{array}{l}\text { proximale } \\
\text { Gliedmaße }\end{array}$} & 8-9 Watterollen & Cast-Foam/synthetische Cast-Watte \\
\hline & 2 Mullbinden & Cast-Filz \\
\hline & 15-20 elastische Bandagen & 7-9 Cast-Binden $(12,5 \mathrm{~cm})$ \\
\hline & 6-7 elastische adhäsive Bandagen & 3-4 Bandagen \\
\hline & Klebeband & 1 elastische adhäsive Bandage \\
\hline & & Klebeband \\
\hline
\end{tabular}

schen der Schienen mit möglicherweise irreversiblen, fatalen Folgen führen.

Der Cast-Verband besteht aus einem dehnbaren Glasfaser- oder Polyestergewebe, das mit Kunststoffharz beschichtet ist ( $\triangleright$ Abb. 1c, 2). Bei Erstkontakt der CastBinde mit Wasser kommt es zur Aktivierung der Bestandteile und die elastische Cast-Binde wird an die vorab gepolsterte Gliedmaße modelliert. Die Aushärtung des Cast-Verbands benötigt in Abhängigkeit vom Material lediglich 15-20 min. Der Cast-Verband hat ein geringes Gewicht und ist meist leichter an die Gliedmaße anzubringen als konventionelle Schienenverbände. Ein Nachteil neben dem hohen Preis ist, dass das Pferd beim Anlegen des Cast-Verbands ruhig stehen muss, um Falten und Rissbildungen zu vermeiden, die sich negativ auf die Biomechanik des Casts auswirken oder Druckstellen hervorrufen können.

Die Polsterung ist bei Schienen- und Cast-Verbänden außerordentlich wichtig, da das traumatisierte Gewebe im Anschluss an das Trauma anschwillt, was zu Komplikationen wie Druckstellen und Strangulationen führen kann. Pferde mit Fraktur- oder Fissurverdacht müssen fachgerecht immobilisiert und stabilisiert werden, da sich eine solche Läsion in eine offene Fraktur mit infauster Prognose verwandeln kann.

\section{Stabilisierung der Gliedmaße(n)}

Für die Notfallstabilisierung einer Fraktur werden die Vorder- und Hintergliedmaßen jeweils in 4 Zonen (Zone I bis IV) eingeteilt ( $\triangleright$ Abb. 3) [2].
Beim Vorderbein reicht

- Zone I vom Huf bis zum distalen Röhrbein,

- Zone II vom distalen Röhrbein bis zum distalen Radius,
- Zone III vom distalen Radius bis zum Ellenbogengelenk und

- Zone IV beinhaltet die Strukturen proximal des Ellenbogengelenks.

\section{Stabilisierung von Zone I}

Ziel der Stabilisierung einer Kronbeinoder Fesselbeinfraktur ist es, die dorsalen Aspekte von Huf-, Kron-, Fessel- und Röhrbein in einer annähernd geraden Linie zu stabilisieren ( $\bullet$ Abb. 2, 4), um dem Risiko einer Dislokation der Fraktursegmente entgegenzuwirken ( $\triangleright$ Abb. 3, 4). Das Vorderbein wird im Bereich des distalen Radius nach kranial aufgehoben ( $\triangleright$ Abb. 2, 4ac). Nach Anlegen des Verbands, der vom Huf bis zum proximalen Metakarpus reicht, wird eine Schiene dorsal angebracht ( $\bullet$ Abb. 4a, b).

Alternativ kann ein Cast-Verband, der den Huf mit einschließt und bis zum proximalen Röhrbein reicht, angebracht werden ( $\bullet$ Abb. 2). Der kommerziell erhältliche Kimzey- oder Monkey-Splint ( $\triangleright$ Abb. 4c) kann nach Anlegen eines Verbands anstelle einer nicht modellierbaren 


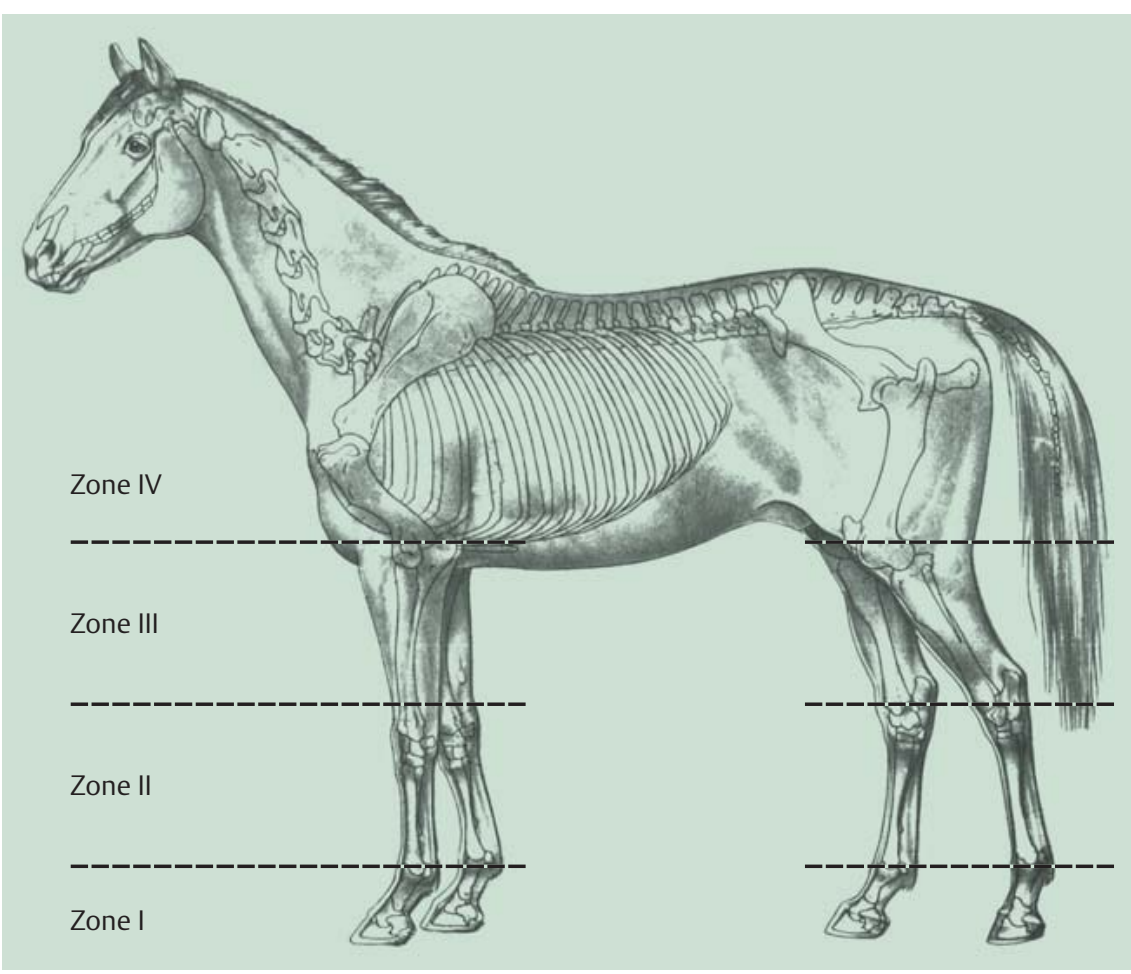

Abb. 3 Einteilung der Gliedmaßen in 4 Zonen zur Notfallstabilisierung (Zone I bis IV; modifiziert nach [2]) Vorderbein: Zone I: Huf bis zum distalen Röhrbein, Zone II: distales Röhrbein bis zum distalen Radius, Zone III: distaler Radius bis zum Ellenbogengelenk, Zone IV: proximal des Ellenbogengelenks. Hinterbein: Zone I: Huf bis zum distalen Röhrbein, Zone II: distales Röhrbein bis zum Sprunggelenk, Zone III: Sprunggelenk bis zum Kniegelenk; Zone IV: proximal des Kniegelenks (modifiziert aus Nickel, Schummer, Seiferle: Lehrbuch der Anatomie der Haustiere. Band 1. 8. Aufl. Parey in MVS Medizinverlage Stuttgart; 2004).

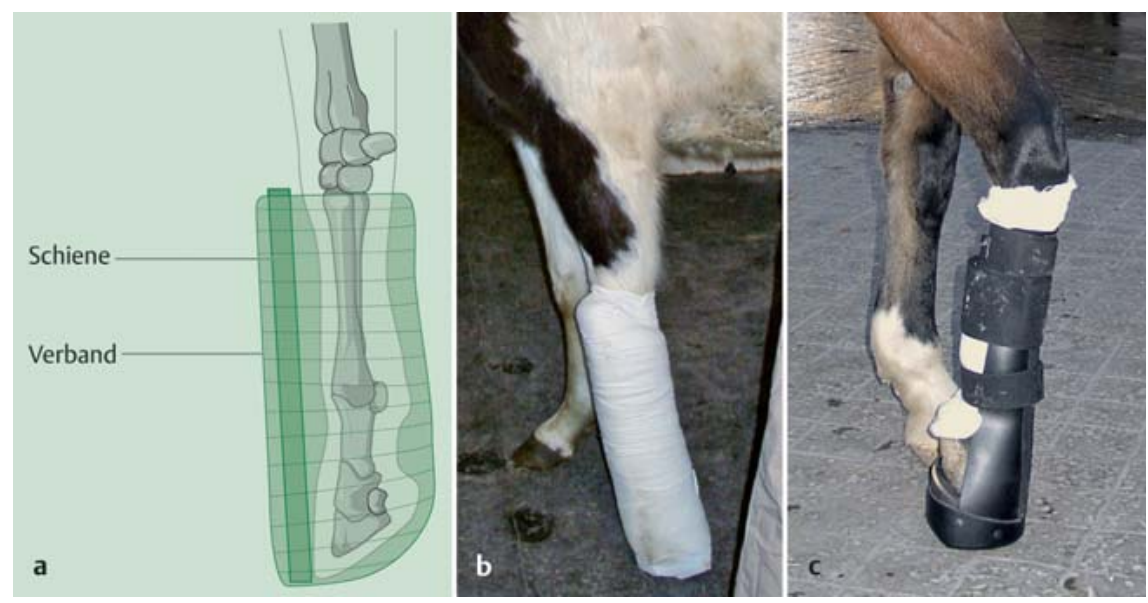

Abb. 4 a bis c Distaler Schienenverband am Vorderbein (Zone I). a Schematisch (modifiziert nach [2]). b Stabilisierung eines Vorderbeins mittels Schienenverband. c Schienung der distalen Gliedmaße mittels Monkey-Splint.

Schiene oder eines Cast-Verbands angebracht werden. Die lateromediale Stabilität der distalen Gliedmaße ist bei der Stabilisierung mittels Kimzey-Splint nicht gegeben, daher sollte dieses Schienungsverfahren nicht bei kondylären Frakturen oder Fissuren des distalen Röhrbeins eingesetzt werden [7].

\section{Stabilisierung von Zone II}

Bei Röhrbeinfrakturen muss eine Stabilisierung mittels Cast- oder Schienenverband vom Huf bis zum Ellenbogengelenk versorgt ( $\bullet$ Abb. 3, 5). Die lateral angebrachte Schiene ist im proximalen Bereich gepolstert und reicht vom Huf bis zur Schulter und verhindert die Abduktion der Gliedmaße $(\bullet$ Abb. 5).

\section{Stabilisierung von Zone IV}

Im Falle der Olecranonfraktur kann es zur Aufhebung der Funktion des M. triceps brachii mit abgesenktem Ellenbogen und gebeugtem Fesselgelenk und Karpalgelenk kommen [9]. Ein bis zum Ellenbogen reichender Verband mit einer kaudalen Schiene bis zum Ellenbogen, erlaubt eine Stabilisierung der Gliedmaße und eine damit assoziierte Verringerung der Angstzustände des Tieres.

Frakturen von Humerus und Skapula bedürfen wegen der umliegenden großen Muskelmassen meist keiner primären Stabilisierung. In ausgewählten Fällen kann ein hoher Verband mit Immobilisierung des Karpus zu einer besseren Gewichtaufnahme und erhöhtem Komfort des Pferdes führen.

\section{Hinterbein}

Beim Hinterbein erstreckt sich

- Zone I vom Huf bis zum distalen Röhrbein,

- Zone II vom distalen Röhrbein bis zum Sprunggelenk,

- Zone III vom Sprunggelenk bis zum Kniegelenk und

- Zone IV über die Strukturen proximal des Kniegelenks.

\section{Stabilisierung von Zone I}

Die Stabilisierung von Zone I am Hinterbein unterscheidet sich wegen des Spannsägemechanismus von der Stabilisierung der Vordergliedmaße. Bei aufgehobener Hinterhand kommt es zur Beugung des Fesselgelenks ( Abb.6). Aus diesem Grund wird die Gliedmaße, nach Anbringung eines Trachtenkeils, an leicht aufgehobener Hintergliedmaße mittels Schienen- oder Cast-Verband stabilisiert. Die Schiene wird dorsal oder plantar angebracht und reicht vom Huf bis zum Kalkaneus.

\section{Stabilisierung von Zone III}

Bei Radiusfrakturen kann es durch Kontraktion der am Bein kraniolateral gelegenen Extensoren zu einer Abduktion im Bereich des Frakturspalts mit medialer Perforation der Haut kommen. Ein hoher Schienenverband vermag dies nicht verhindern. Aus diesem Grund werden Radiusfrakturen mittels bis zum Ellenbogen reichendem Schienen- oder Cast-Verband

\section{Stabilisierung von Zone II}

Frakturen des Metatarsus' werden mittels eines hohen Cast-Verbands oder eines Schienenverbands mit einer lateralen Schiene, die vom Huf bis zum Kniegelenk reicht, stabilisiert. Hohe Cast- und Schienenverbände bergen die Gefahr des Hinfallens mit Ruptur des M. peroneus tertius 


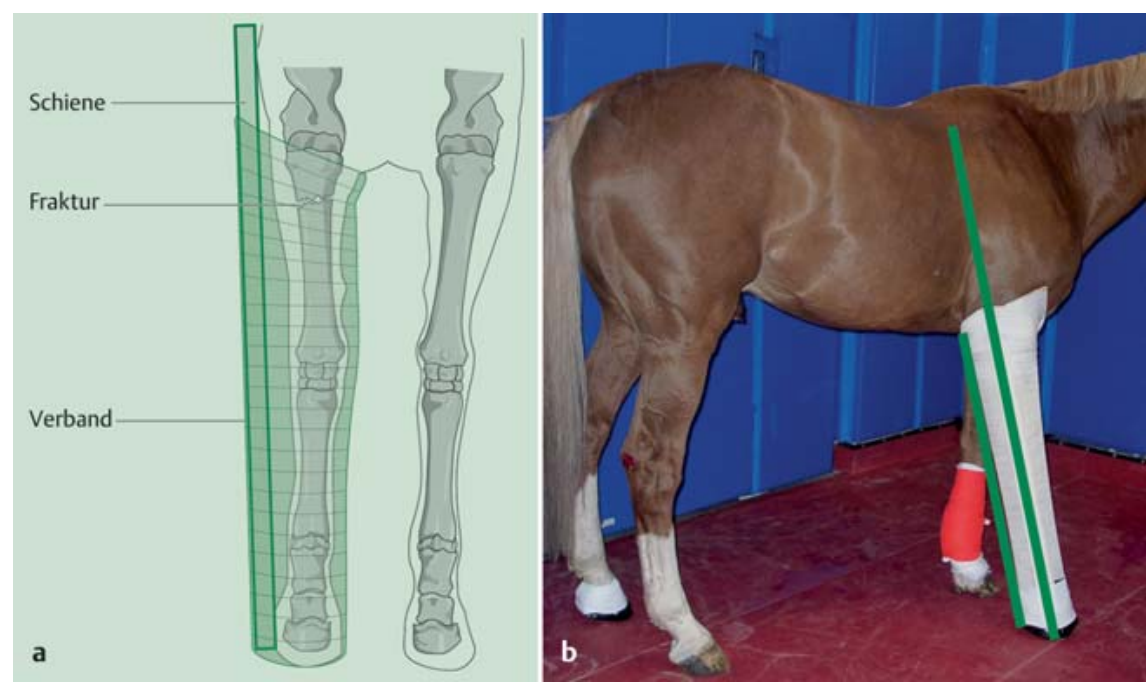

Abb. 5 a und b Hoher Schienenverband am Vorderbein (Zone III). a Schematisch (modifiziert nach [2]). b Stabilisierung einer distalen Radiusfraktur mittels eines hohen Verbands sowie kaudaler und lateraler Schiene.

oder einer Hüftgelenksluxation oder Femurfraktur.

\section{Stabilisierung von Zone III}

Tibiafrakturen sind wegen des Spannsägemechanismus schwierig zu stabilisieren. Der Cast-Verband sowie auch der Schienenverband reichen vom Huf bis zum Kniegelenk. Die Schiene reicht vom Huf bis zum Tuber coxae und wird lateral befestigt ( $\triangleright$ Abb. 7). Diese Stabilisierung soll einer Abduktion entgegenwirken und eine Verletzung der Haut im medialen Bereich der Tibia verhindern.

\section{Stabilisierung von Zone IV}

Femurfrakturen können nicht mittels Verband oder Schienen stabilisiert werden. Der Femur ist jedoch von einer großen Muskelmasse umgeben.

\section{Transport zur Klinik}

Der Transport eines Pferdes mit Fraktur oder Frakturverdacht sollte in einem genügend großem Pferdeanhänger, möglichst mit Vorderrampe erfolgen [7]. Zum Ein- und Ausladen des Pferdes sollte die Verladerampe keine Steigung aufweisen. Starke Beschleunigungs- und Bremsmanöver sollten vermieden werden, wobei Beschleunigungsmanöver im Gegensatz zu Bremsmanövern im Regelfall genauer dosiert werden können [7].

Es gibt Transportfahrzeuge, die für den Transport von Notfallpferden konstruiert wurden und über spezielle Einrichtungen wie z.B. Vorderausstiegsrampe, Seilwinden oder Gurte verfügen. Pferde mit einer Fraktur der Vordergliedmaße sollten, wenn möglich, in Spezialhängern mit dem Kopf gegen die Fahrtrichtung transportiert werden, damit bei unvorhersehbaren Bremsmanövern die Gewichtaufnahme auf den gesunden Hintergliedmaßen erfolgen kann [2]. Bei Frakturen der Hintergliedmaße wird das Pferd möglichst mit dem Kopf in Fahrtrichtung transportiert. Bei normalen Transportanhängern ist es wichtig, dass der Frakturpatient in Fahrtrichtung so angebunden wird, das Kopf und Hals frei beweglich sind, damit sich das Pferd besser ausbalancieren kann. Eine besondere Bedeutung kommt der Bruststange zu, die für die Stabilisierung des Pferdes von Wichtigkeit ist. Ein Breitstellen des Pferdes oder ein Herausnehmen der Mitteltrennwand erlaubt es dem Pferd, sich besser im normalen Transportanhänger auszubalancieren (Rattenhuber, persönliche Mitteilung). Ausgebildete 


\section{ps.fachspiegel}

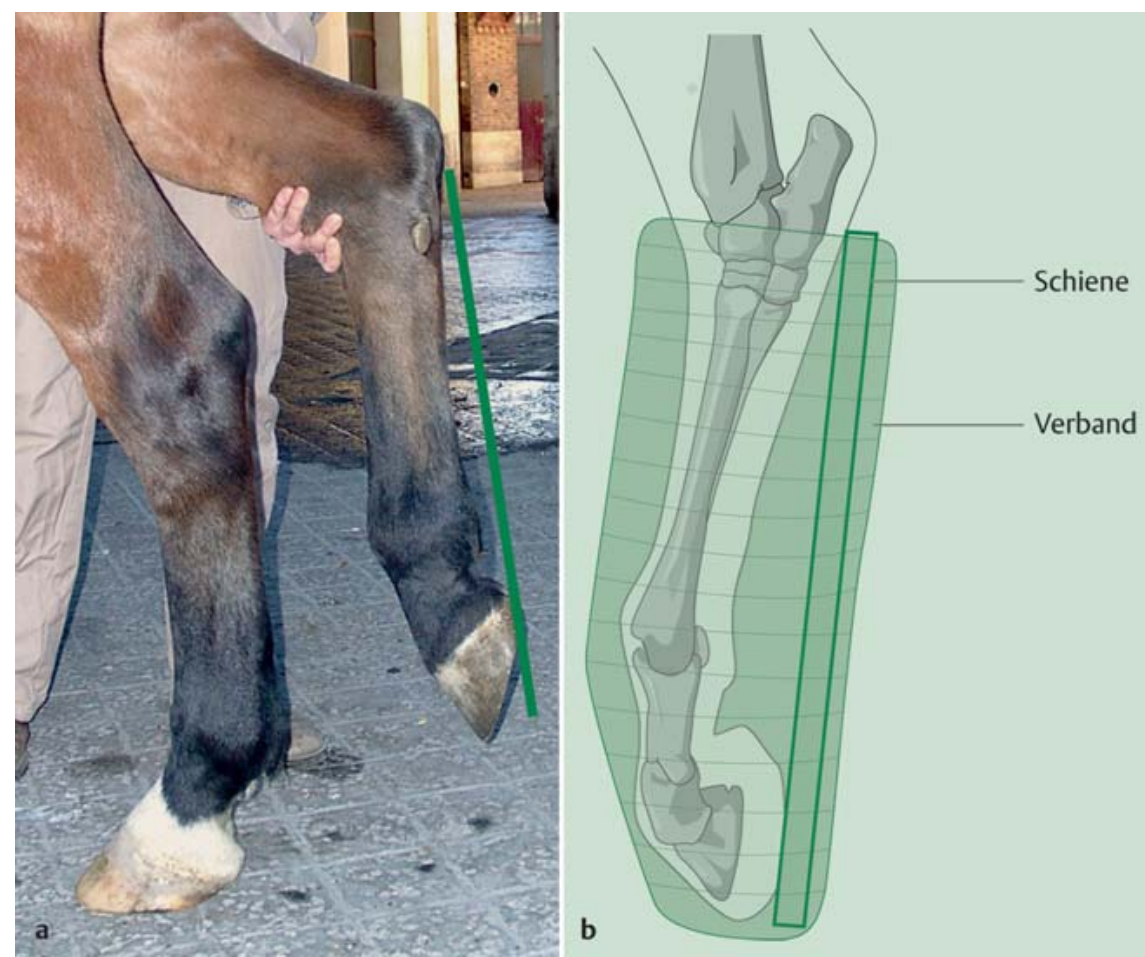

Abb. $\mathbf{6}$ a und $\mathbf{b}$ a Bei aufgehobener Hintergliedmaße kommt es aufgrund des Spannsägemechanismus zur Beugung des Fesselgelenks. b Stabilisierung eines Hinterbeins mittels distalem Schienenverband (Zone I; modifiziert nach [2])
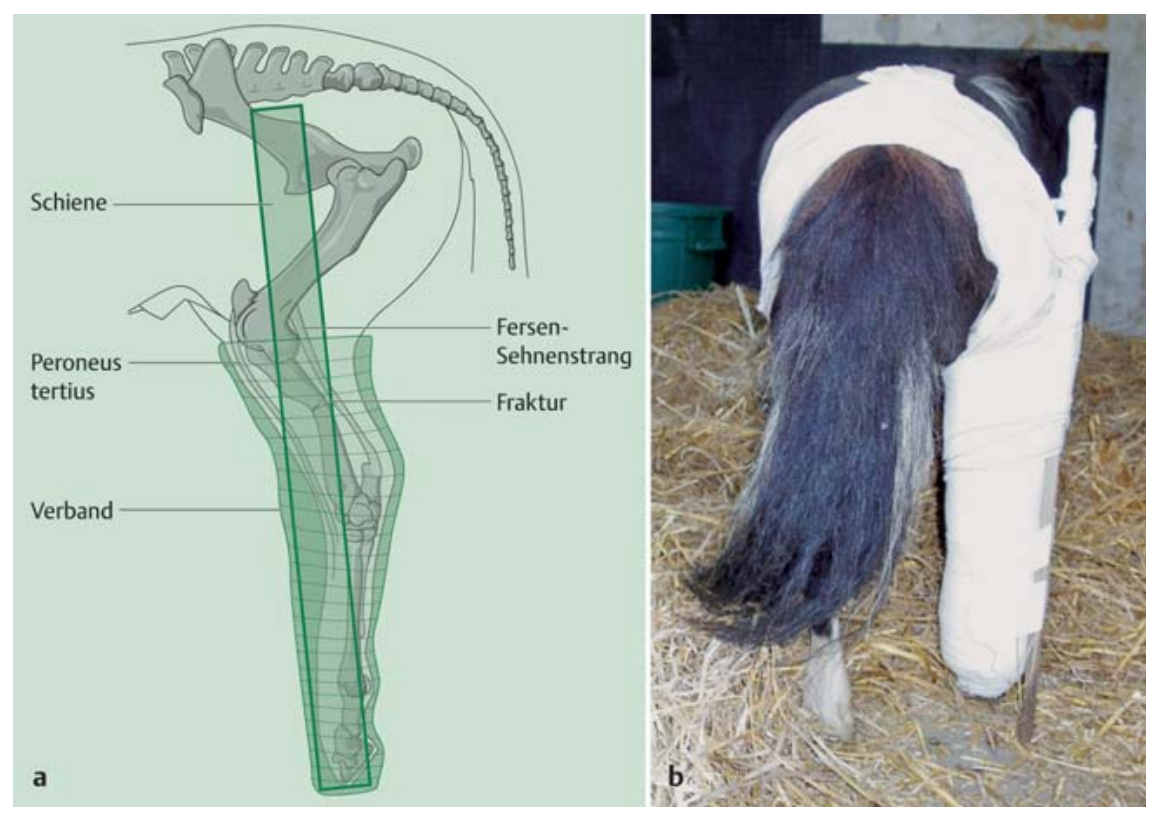

Abb. 7 a und b Hoher Schienenverband am Hinterbein (Zone III). a Schematische Ansicht (modifiziert nach [2]) einer Stabilisierung mittels lateraler Schiene. b Stabilisierung einer distalen Tibiafraktur mittels eines hohen Verbands und lateraler Holzschiene.

Großtierrettungsteams sind derzeit in einigen europäischen (z.B. Liechtenstein, Schweiz) und nordamerikanischen Ländern vorhanden [5].

\section{Key words}

horse - fracture - emergency therapy transport ment of Emergencies at Equine Competitions. Newmarket, Suffolk, UK: British Equine Veterinary Association; 1996: 21-28

4 Carter BG, Schneider RK, Hardy J, Bramlage LR, Bertone AL. Assessment and treatment of equine humeral fractures: retrospective study of 54 cases (1972-1990). Equine Vet J 1993; 25: 203-207

5 Fürst AE, Jackson M, Keller R, Von Salis B. Notfallversorgung und Transport des Frakturpferdes. Pferdeheilkunde 2009; 4: 302-309

6 Hardy J, Burkhardt HA, Beard W. Equine emergency and intensive care: case survey and assessment of needs (1992-1994). In Proc AAEP 1996; 42: 182-183

7 Mudge MC, Bramlage LR. Field fracture management. Vet Clin Equine 2006; 23: 117-133

8 Nixon AJ. General considerations in selecting cases for fracture repair. In: Nixon AJ, ed. Equine Fracture Repair. Philadelphia, PA, USA: W. B. Saunders; 1996: 30-35.

9 Nixon AJ. Fractures of the ulna. In: Nixon AJ, ed. Equine Fracture Repair. Philadelphia, PA, USA: W. B. Saunders; 1996: 231-241.

10 Sweeney CR, Hodge TG. Sudden death in a horse following fracture of the acetabulum and iliac artery laceration. J Am Vet Med Assoc 1983, 182: 712-713

11 Walmsley J. Management of a suspected fracture. In: Dyson S, ed. A Guide to the Management of Emergencies at Equine Competitions. Newmarket, Suffolk, UK: British Equine Veterinary Association; 1996: 13-20

12 Zekas LJ, Bramlage LR, Embertson RM, Hance SR. Results of treatment of 145 fractures of the third metacarpal/metatarsal condyles in 135 horses (1986-1994). Equine Vet J 1999; 31: 309-313

\section{Online}

http://dx.doi.org/10.1055/s-0030-1250254

\section{PD Dr. Bianca Carstanjen, PhD}

Department of Large Animal Disease

Faculty of Veterinary Medicine

Warsaw University of Life Science

Nowoursynowska 100

02-786 Warschau

Polen

bcarstanjen@web.de

\section{Dr. Stefan Rattenhuber}

Tierklinik Seehof - Pferde Physiotherapie Centrum Seehof 2

86911 Dießen

info@tierklinikseehof.de 